\title{
Synthesis of organic liquid crystals containing selectively fluorinated cyclopropanes
}

\author{
Zeguo Fang ${ }^{* 1}$, Nawaf Al-Maharik ${ }^{1,2}$, Peer Kirsch ${ }^{3}$, Matthias Bremer ${ }^{3}$, \\ Alexandra M. Z. Slawin ${ }^{* 1}$ and David O'Hagan*1
}

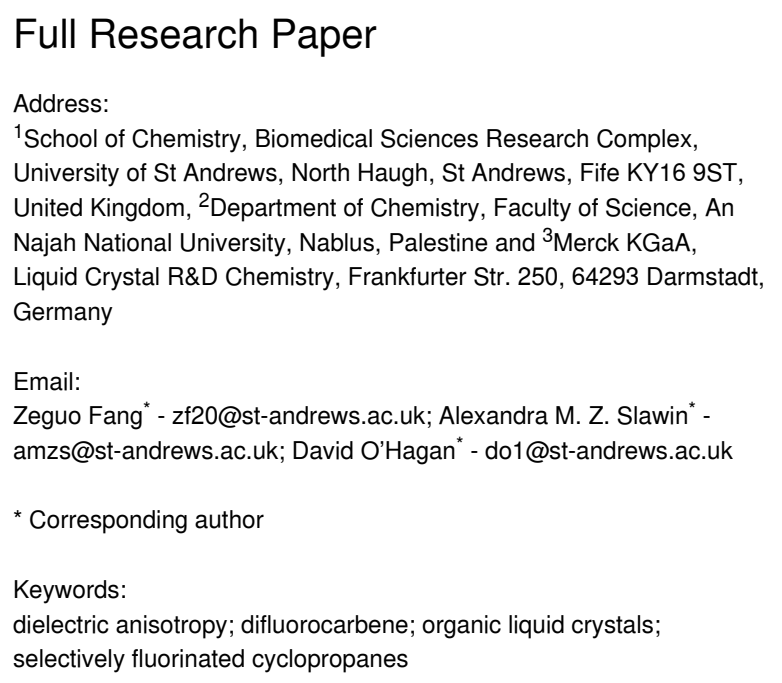

Beilstein J. Org. Chem. 2020, 16, 674-680. doi:10.3762/bjoc.16.65

Received: 08 February 2020

Accepted: 03 April 2020

Published: 14 April 2020

This article is part of the thematic issue "Organo-fluorine chemistry V".

Associate Editor: P. J. Skabara

(c) 2020 Fang et al.; licensee Beilstein-Institut. License and terms: see end of document.

\begin{abstract}
This paper describes the synthesis of a series of organic liquid crystals (LCs) containing selectively fluorinated cyclopropanes at their termini. The syntheses used difluorocarbene additions to olefin precursors, an approach which proved straightforward such that these liquid crystal candidates could be efficiently prepared. Their physical and thermodynamic properties were evaluated and depending on individual structures, they either displayed positive or negative dielectric anisotropy. The study gives some guidance into effective structure-property relationships for the design of LCs containing selectively fluorinated cyclopropane motifs.
\end{abstract}

\section{Introduction}

Display materials associated with modern electronic devices such as personal mobile phones and TV sets, have changed dramatically in the past decades as liquid crystal display (LCD) technology has evolved $[1,2]$. A key challenge is to make suitable liquid crystalline (LC) materials that satisfy the requirements for different LCD technologies. For the traditional twisted nematic (TN) LCD technology, devices require liquid crystals with display positive dielectric anisotropy by which the molecular dipole moment is oriented parallel to the long axis of the molecule, while for the current vertical alignment (VA) LCD technology, liquid crystals with negative dielectric anisotropy are required [3]. Fluorine, as the most electronegative atom, forms stable bonds to carbon and can thus induce polarity. It is also attractive as a design feature due to the low polarizability of the C-F bond relative to other polar substituents such as nitrile and ester groups, and this reduces intermo- 
lecular interactions leading to lower viscosities and thus increased life time reliability [4,5]. A significant effort has been devoted to the development of liquid crystals with either positive or negative dielectric anisotropy by introducing the $\mathrm{C}-\mathrm{F}$ bond or the dipole of a $\mathrm{CF}_{2}$ group either parallel or perpendicular to the long molecular axis. For example, in the area of negative liquid crystalline materials, Kirsch et al. have reported the synthesis of bicyclohexane and bis(cyclohexyl)ethane liquid crystals which contain axial fluorine substituents (Figure 1, 1 and 2), however, due to the propensity for HF elimination from these molecules over time, these liquid crystals have not been adopted as commercial products [6,7]. 2,3-Difluoroaryl motifs such as $\mathbf{3}$ have proven to be a successful class of negative dielectric anisotropic liquid crystals and the more rigid 1,1,6,7tetrafluoroindane 4 led to an enhanced polarity and improved negative dielectric anisotropy character [8]. Other liquid crystals of this class are the recently reported fluorinated cyclohexane 5 and 1,1,2,2-tetrafluorocyclohexane 6 [9,10], but the complexity of their synthesis has limited their development as practical materials.

As a consequence, it continues to be a research objective to introduce polar substituents in a manner that can influence the nature of the dielectric anisotropy of the resultant material. In this context we explore the cyclopropane motif containing fluorine atoms. Selectively fluorinated cyclopropanes have been widely used in pharmaceutical research [11], however, the introduction of fluorinated cyclopropanes into liquid crystal scaffolds has not received much attention. Haufe et al. [12], reported enantiomerically pure fluorinated diphenylcyclopropane carboxylates as potential chiral dopants to induce a cholesteric phase, but this was not extended to the exploration of positive or negative dielectrics. Recently, we reported the efficient synthesis of $\alpha, \beta, \beta$-trifluorocyclopropanes 7 (Figure 2) through difluorocarbene addition to alpha-fluorostyrenes [13]. This used the extraordinary useful method for the generation of difluorocarbene from the Ruppert-Prakash $\left(\mathrm{TMSCF}_{3}\right)$ reagent [14]. DFT analysis suggests the lowest energy conformer of aryl $\alpha, \beta, \beta$-trifluorocyclopropane 7 orients the $\mathrm{C}-\mathrm{F}$ bond perpendicular to the aryl ring. So, if the $\alpha, \beta, \beta$-trifluorocyclopropane motif were introduced into an appropriate LC scaffold, it could reasonably induce negative dielectric anisotropy if the dipole associated with the cyclopropane remains oriented perpendicular to the molecular axis. Based on this idea, we report the design and synthesis of cyclopropanes $\mathbf{8}$ and $\mathbf{9}$ with an objective to assess their LC properties. Cyclopropane $\mathbf{8}$ with a single $\mathrm{CF}_{2}$ group offered a control to assess the influence of the alphafluorine atom in cyclopropane $\mathbf{9}$ containing three fluorines. In order to extend the study to explore other selectively fluorinated cyclopropane motifs we set out to prepare liquid crystal candidates 10 and isomers 11a and 11b. Ether 10 should have significant rotational freedom due to rotation around the ether bonds and will be compared with isomers 11a and 11b which are much more conformationally constrained (Figure 2).

Therefore, the aim of the study was to prepare these target liquid crystals and evaluate their properties relative to each other, to establish if there were any obvious structure-activity relationships to emerge for the design of positive or negative dielectric materials. Also the fixed spiro $\mathrm{CF}_{2}$-containing cyclo-

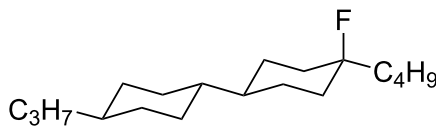

$1 \Delta \varepsilon=-1.7$

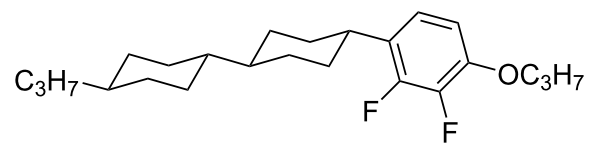

$3 \Delta \varepsilon=-5.4$

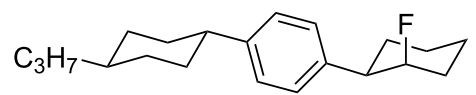

$5 \Delta \varepsilon=-1.2$<smiles>C=CCCC1(F)CCC(CCC2(F)CCC(C=C)CC2)CC1</smiles>

$2 \Delta \varepsilon=-3.8$

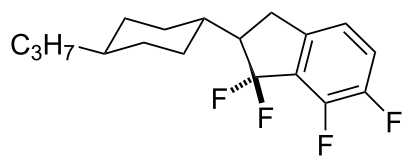

$4 \Delta \varepsilon=-8.6$

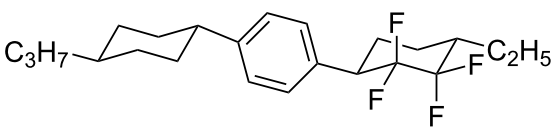

$6 \Delta \varepsilon=-9.4$

Figure 1: Examples of liquid crystal candidates with negative values for dielectric anisotropy $(\Delta \varepsilon)[6-10]$. 


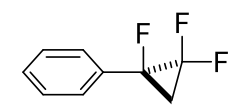

7

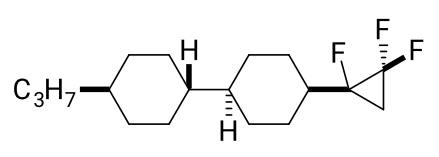

9

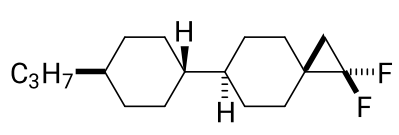

$11 \mathrm{a}$

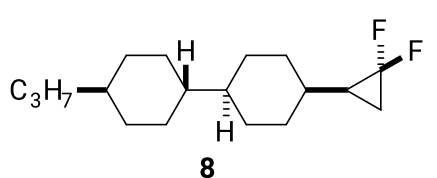

8

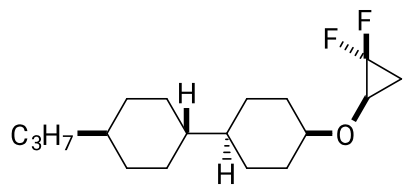

10

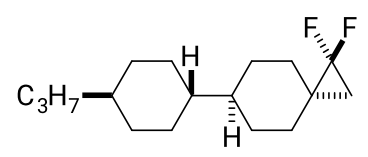

11b

Figure 2: Synthetic candidate LC targets 8-11.

propanes 11a and 11b became targets to assess the impact of a configurationally rigid bicyclic motif. It is relatively obvious that for each of these isomers the $\mathrm{CF}_{2}$ dipole in each cyclopropane is orientated either along the molecular axis (for 11a) or perpendicular to the molecular axis (for 11b), and these were anticipated at the outset to give positive and negative dielectric anisotropy values, respectively.

\section{Results and Discussion}

\section{Synthesis of liquid crystal candidates 8-11}

The synthesis of cyclopropane $\mathbf{8}$ started from olefin $\mathbf{1 2}$ (supplied by Merck KGaA, Darmstadt), as illustrated in Scheme 1. Difluorocyclopropane $\mathbf{8}$ can be directly prepared through a $[2+1]$ carbene cycloaddition reaction. Thus, treatment of 12 with trimethyl(trifluoromethyl)silane $\left(\mathrm{TMSCF}_{3}\right)$ and sodium iodide under refluxing conditions $[13,14]$ gave the corresponding product $\mathbf{8}$ in one step and a $55 \%$ yield.

The synthesis of trifluorocyclopropane $\mathbf{9}$ is shown in Scheme 2. Compound 9 was prepared through bromofluorination of $\mathbf{1 2}$, followed by base-induced $\mathrm{HBr}$ elimination [15], and then difluorocarbene addition to generate $\mathbf{9}$. The starting olefin $\mathbf{1 2}$ was exposed to an excess of $N$-bromosuccinimide (NBS) and HF.Py to generate $\mathbf{1 3} \mathbf{a}$ and $\mathbf{1 3 b}$ as a mixture of regioisomers in a ratio of $4: 1$. There was no requirement to separate these isomers at this stage. Subsequent addition of potassium tert-butoxide into a mixture of 13a and 13b in dichloromethane (DCM) resulted in an efficient elimination to generate vinyl fluoride $\mathbf{1 4}$ as a major product. Vinyl fluoride 14 was purified by column chromatography in a $42 \%$ yield over the two steps. Finally, difluorocarbenene (: $\left.\mathrm{CF}_{2}\right)$ addition using the $\mathrm{TMSCF}_{3} / \mathrm{NaI}$ protocol was used to generate the target product 9 in $46 \%$ yield.

The preparation of cyclopropyl ether $\mathbf{1 0}$ was accomplished as illustrated in Scheme 3. Reduction of cyclohexanone 15 with $\mathrm{NaBH}_{4}$ gave cyclohexanol 16 in a ratio of 2:1. The major trans product 16a was purified as a single entity by column chromatography and was isolated in $45 \%$ yield. Vinyl ether $\mathbf{1 7}$ could be efficiently prepared using the methodology developed by Bosch [16]. Accordingly, treatment of $\mathbf{1 6 a}$ with butyl vinyl ether, bathophenanthroline (BPhen) and $\mathrm{Et}_{3} \mathrm{~N}$, using palladium(II) trifluoroacetate as a catalyst, gave vinyl ether 17 in $62 \%$ yield in a single step. Finally, difluorocarbene-mediated difluorocyclopropanation generated $\mathbf{1 0}$ in $50 \%$ yield.

The synthesis of the diastereoisomers of spiro LC candidates 11a and 11b also started from ketone $\mathbf{1 5}$ as illustrated in Scheme 4. Treatment of ketone $\mathbf{1 5}$ with methylenetriphenyl-

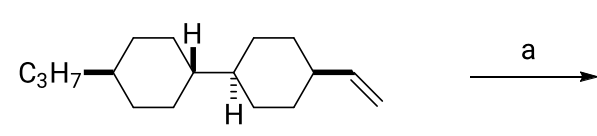

12

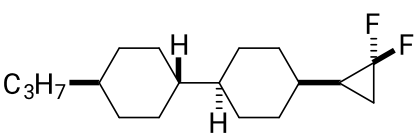

8 


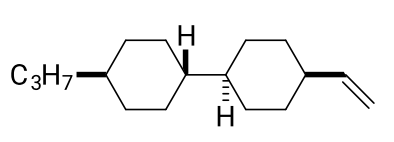

12
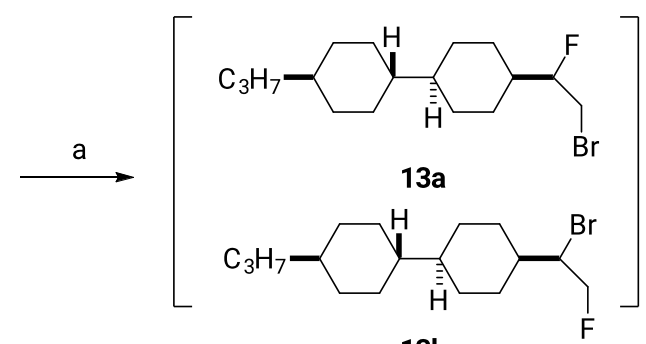

$13 b$

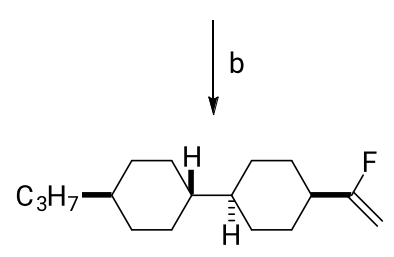

14

Scheme 2: Synthesis of 9. Reagents and conditions: a) NBS, HF·Py, DCM; b) $t$-BuOK, DCM, 42\% in two steps [15]; c) TMSCF 3 , Nal, THF, reflux, $46 \%$.

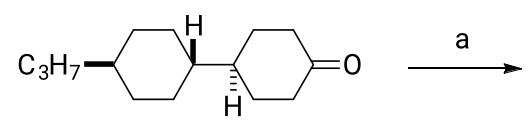

15

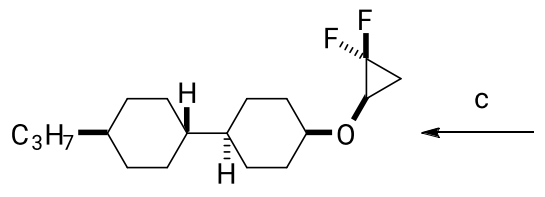

10<smiles></smiles>

$16 \mathbf{a}$

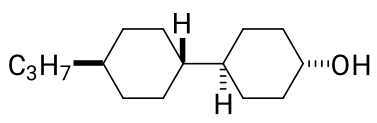

$16 \mathrm{~b}$

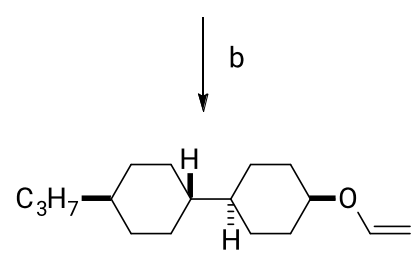

17

Scheme 3: Synthesis of compound 10. Reagents and conditions: a) $\mathrm{NaBH}_{4}, \mathrm{MeOH}, \mathrm{rt}, 45 \%$; b) $\mathrm{C}_{4} \mathrm{H}_{9} \mathrm{OCH}=\mathrm{CH}_{2}, \mathrm{Pd}(\mathrm{TFA})_{2}, \mathrm{BPhen}, \mathrm{Et}{ }_{3} \mathrm{~N}, 75{ }^{\circ} \mathrm{C}, 62 \%$ [16]; c) $\mathrm{TMSCF}_{3}$, Nal, THF, reflux, $50 \%$.

phosphine which was generated in situ from methyltriphenylphosphonium bromide $\left(\mathrm{PPh}_{3} \mathrm{CH}_{3} \mathrm{Br}\right)$, generated exo-methylene cyclohexane $\mathbf{1 8}$ in a very straightforward manner, and the product could be isolated by chromatography in $72 \%$ yield [17]. This olefin was then subject to a difluorocarbene cyclopropanation using $\mathrm{TMSCF}_{3}$ and $\mathrm{NaI}$, and this generated both isomers, 11a and 11b, in a ratio of 5:1, respectively. After three recrystallizations, the major isomer 11a could be isolated as a single stereoisomer. It proved impossible to isolate a sample of isomer 11b without a significant and dominant contamination with 11a. Thus, it was with some frustration that we were unable to prepare a suitable sample, both in quantity and purity, of isomer 11b for subsequent analysis. Stereoisomer 11a was a crystalline solid and a suitable crystal was subject to X-ray structure analysis and the structure is shown in Scheme 4. In this way the stereochemistry of $\mathbf{1 1 a}$ and $\mathbf{1 1 b}$ could be confirmed unambiguously.

\section{Evaluation of liquid crystal candidates 8-11a}

With the four difluorocyclopropanes 8-11a in hand, our attention focused on an experimental evaluation of their thermodynamic and physical properties, such as birefringence $(\Delta n)$ and dielectric anisotropy $(\Delta \varepsilon)$. DSC and POM analysis (Table 1) indicated that only compounds $\mathbf{8}$ and $\mathbf{9}$ exhibited smectic phases. 
<smiles>[14CH3]C1CCC(C2CCC(=O)CC2)CC1</smiles>

15

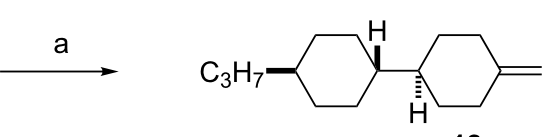

18

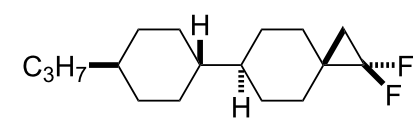

$11 \mathrm{a}$
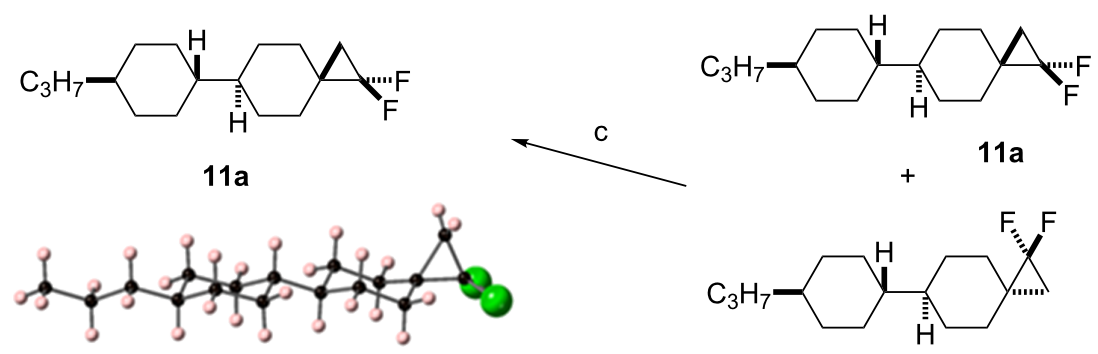

$+\quad 11 a$

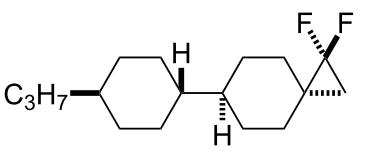

$11 b$

Scheme 4: Synthesis of compounds 11. Reagents and conditions: a) $\mathrm{PPh}_{3} \mathrm{CH}_{3} \mathrm{Br}, t$-BuOK, diethyl ether, $0{ }^{\circ} \mathrm{C}$ to rt, $72 \%$ [17]; b) $\mathrm{TMSCF}$, $\mathrm{Nal}$, $\mathrm{THF}$, reflux; c) recrystallization, $27 \%$.

The smectic phase of LC $\mathbf{8}$ was maintained over a broad temperature range $\left(54-84^{\circ} \mathrm{C}\right)$, whereas that for 10 and $\mathbf{1 1 a}$ only showed a melting point and these compounds did not display any obvious liquid crystallinity (Table 1 ). Therefore, any development of these latter materials would require extended core structures to enhance their LC potential.

Table 1: Phase transition temperatures of compounds 8-11.

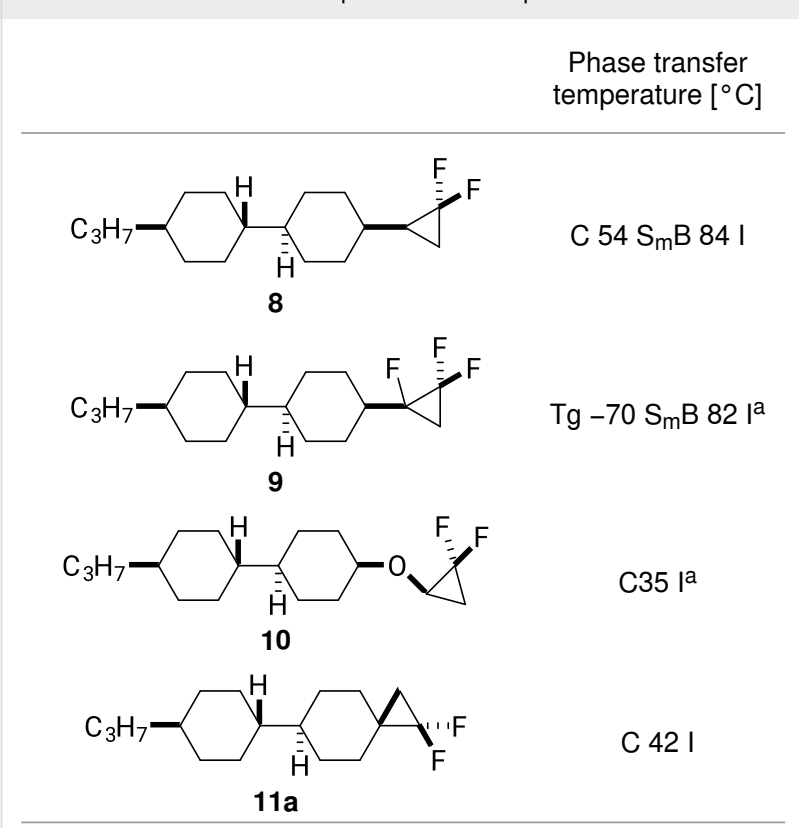

aDecomposition $\left(C=\right.$ crystalline, $S_{m} B=$ smectic $B, I=$ isotropic, $\mathrm{Tg}=$ glass transition temperature).

The birefringence $\Delta n$ (Table 2) for compounds 8-11a was measured (Abbe refractometer). In all cases the $\Delta n$ values were low, which is entirely consistent with their core aliphatic structures, unlike materials with aromatic rings. The differences for $\Delta n$ for $\mathbf{8 , 9}$ and $\mathbf{1 0}$, were found to be small, whereas the spirocyclopropane 11a had a significantly, decreased $\Delta n$.

\begin{tabular}{lll} 
Table 2: Physical properties of compounds 8-11. \\
Compound & $\Delta n$ & $\Delta \varepsilon$ \\
\hline $\mathbf{8}^{\mathrm{a}}$ & 0.056 & -0.5 \\
$\mathbf{9}^{\mathrm{b}}$ & 0.055 & -0.2 \\
$\mathbf{1 0}$ & 0.063 & 1.3 \\
$\mathbf{1 1}$ & 0.036 & 4.8 \\
$\mathbf{1 1 a ^ { \mathrm { c } }}$ & 0.054 & 4.3 \\
$\mathbf{1 1 \mathbf { b } ^ { \mathrm { c } }}$ & 0.051 & -1.1 \\
\hline
\end{tabular}

${ }^{a} \Delta n$ were extrapolated from a $10 \mathrm{wt} \%$ solution in liquid crystal mixture Merck ZLI-4792. $\Delta \varepsilon$ were extrapolated from a $10 \mathrm{wt} \%$ solution in liquid crystal mixture Merck Merck ZLI-2857. ${ }^{b} \Delta n$ were extrapolated from a 5 wt \% solution in liquid crystal mixture Merck ZLI-4792. $\Delta \varepsilon$ were extrapolated from a $10 \mathrm{wt} \%$ solution in liquid crystal mixture Merck Merck ZLI-2857. ' Calculated data only at AM1//B3LYP/6-31G(d) level [18].

As discussed, either positive or negative dielectric anisotropy is an important parameter related to the driving voltage of LC displays and in order to reduce energy consumption, good materials should induce a large value for dielectric anisotropy. Compounds 8 and $\mathbf{9}$ were found to exhibit the expected negative $\Delta \varepsilon$ values, however, the overall polarity is low $(\mathbf{8}=-0.5,9=-0.2)$. This is presumably due to the ability of the $\mathrm{C}-\mathrm{C}$ bond attached to cyclopropane to rotate, and thus the compound will adopt conformations with low overall molecular polarities. A comparison of the $\Delta \varepsilon$ for $\mathbf{8}$ and $\mathbf{9}$ illustrates that incorporation of the third alpha-fluorine lowers the overall $\Delta \varepsilon$ value. This is presum- 
ably due to a resulting decrease in the overall molecular dipole moment $\left(\mu_{\perp}\right)$ along the minor axis. When an ether was used as the linkage in compound $\mathbf{1 0}$, the compound becomes more flexible and, perhaps surprisingly, the overall $\Delta \varepsilon$ parameter became more positive. Clearly the effect of introducing a $\mathrm{C}-\mathrm{O}$ bond renders this molecule dissimilar from the others in this series. However, changing the core aliphatic structure to the more rigid spiro structure 11a, brought about a dramatic enhancement of the molecular dipole moment $\left(\mu_{\|}\right)$, resulting in a much more positive and increased parameter value $(\Delta \varepsilon=4.8)$. This experimental value was close to the predicted calculated value $(\Delta \varepsilon=4.3)$ as shown in Table 2. It is clear that there is an advantage in a rigid structure for imparting a maximum polarity. As stated above, we were unable to obtain a stereochemically pure sample of diastereoisomer 11b, free from 11a, however, theory indicates that $\mathbf{1 1 b}$ should display negative dielectric anisotropy, although the magnitude of the parameter was relatively low $(\Delta \varepsilon=-1.1)$. Thus the two isomers constitute two different classes of LC materials, entirely as a consequence of stereochemistry.

The best compound of this series is 11a and it was of interest to get some insight into its conformational flexibility. Thus, a conformational analysis of 11a (and 11b) was conducted computationally $(\omega \mathrm{B} 97 \mathrm{X}-\mathrm{D} / 6-311+\mathrm{G}(2 \mathrm{~d}, \mathrm{p}) / / \omega \mathrm{B} 97 \mathrm{X}-\mathrm{D} / 6-31 \mathrm{G}(\mathrm{d})+\mathrm{ZPE}$ level (see Supporting Information File 1)). There are many potential low energy conformations arising from alkyl side chain $\mathrm{C}-\mathrm{C}$ bond rotations which would not be anticipated to change the overall properties significantly, however, we were interested in assessing the energy required for the bispirocyclohexane ring to adopt an axial rather than the lower energy equatorial arrangement. For 11a, the lowest energy conformer found was a rotamer around the central $\mathrm{C}-\mathrm{C}$ bond linking the cyclohexane rings, but an essentially isoenergetic conformer is that found in the X-ray crystal structure. The first conformers where the terminal bispirocyclohexane ring lies axial are significantly higher in energy $\left(\Delta G=+1.25 \mathrm{kcal} / \mathrm{mol}^{-1}\right.$ and $\Delta G=+1.19 \mathrm{kcal} / \mathrm{mol}^{-1}$ ) than the equatorial conformers as illustrated in Figure 3. Notably for both the axial and equatorial conformers, the orientation of the $\mathrm{CF}_{2}$ group, and therefore its dipole, remains perpendicular to the long molecular axis (requirement for + ve dielectric). A similar result was obtained for stereoisomer 11b (see Supporting Information File 1) again with the first axial conformer at $\Delta G=+1.14 \mathrm{kcal} / \mathrm{mol}^{-1}$ above the ground state equatorial conformer. In this case the orientation of the $\mathrm{CF}_{2}$ group, and therefore the dipole, remains perpendicular to the long molecular axis (requirement for -ve dielectric) for both the axial and equatorial conformers. This analysis, and that of the X-ray structure, suggests that the equatorial conformers will dominate in condensed phases, and that the overall molecular dipoles will not be compromised by minor contributions of axial conformers.

\section{Conclusion}

In conclusion, a series of candidate liquid crystals 8-11a with difluorocyclopropane motifs at their termini were prepared in a straightforward manner. In all cases the cyclopropane was introduced by difluorocarbene addition to an olefin, the difluorocar-

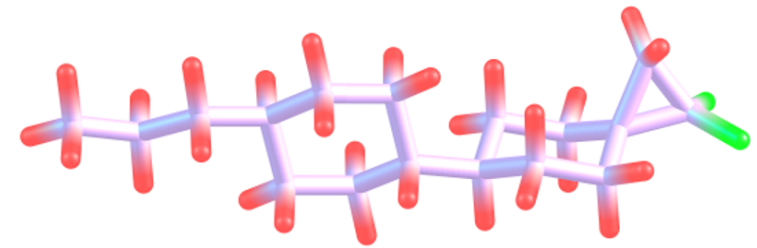

$\Delta G=0.00 \mathrm{kcal} \mathrm{mol}-1$

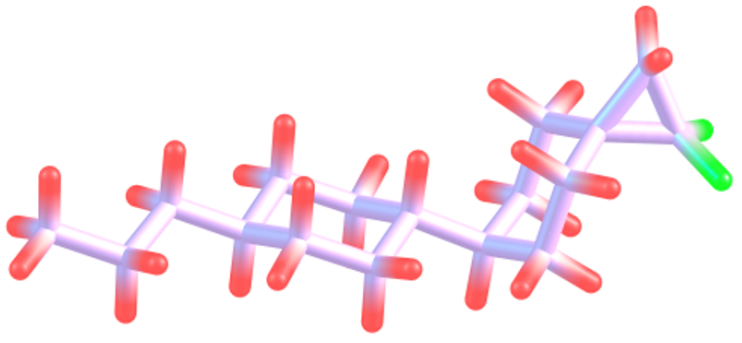

$\Delta G=+1.25 \mathrm{kcal} \mathrm{mol}^{-1}$

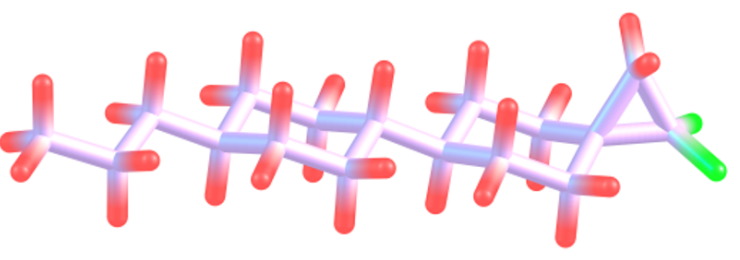

$\Delta G=0.10 \mathrm{kcal} \mathrm{mol}^{-1}$ (close to $\mathrm{X}$-ray structure)

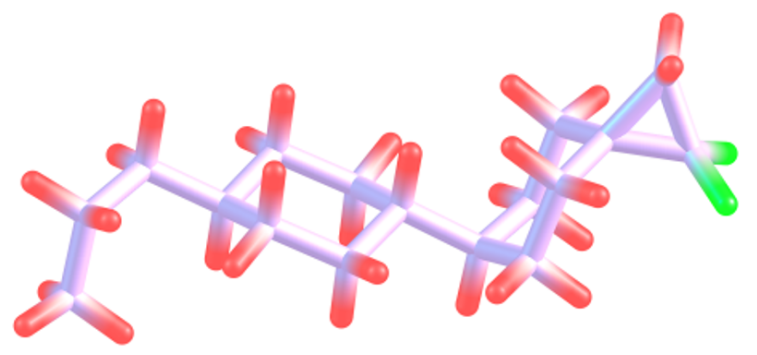

$\Delta G=+1.19 \mathrm{kcal} \mathrm{mol}^{-1}$

Figure 3: Theory study exploring the relative energies for different conformers of 11a. 
bene being generated from $\mathrm{TMSCF}_{3} / \mathrm{NaI}$. An evaluation of the physical properties of LC candidates 8-11a revealed that LC's 8 and 9 exhibited smectic phases with low levels of negative dielectric anisotropy. The relatively high positive dielectric anisotropy of compound 11a indicates that the configurational constrained motif is required to increase the magnitude of the $\Delta \varepsilon$ parameter. Although it proved challenging to isolate a stereochemically clean sample of isomer $\mathbf{1 1 b}$ for experimental evaluation, theory predicts that it will have a modest negative dielectric value, indicating the importance of stereochemistry in dictating the class of LC material. We conclude too that the overall performance of prototype LCs 11a and 11b should not be compromised by conformational flexibility. Although none of the compounds prepared here met all of the criteria for commercial development, the study gives an insight into the potential for this novel difluorocyclopropane motif in liquid crystal design and development.

\section{Supporting Information}

\section{Supporting Information File 1}

Experimental protocols and NMR spectra.

[https://www.beilstein-journals.org/bjoc/content/

supplementary/1860-5397-16-65-S1.pdf]

\section{Supporting Information File 2}

Crystallographic information file of compound 11a.

[https://www.beilstein-journals.org/bjoc/content/

supplementary/1860-5397-16-65-S2.cif]

\section{Acknowledgements}

The EPSRC Mass Spectroscopy Service at Swansea is acknowledged for analysis.

\section{Funding}

We thank the Chinese Scholarship Council for a studentship (ZF).

\section{ORCID ${ }^{\circledR}$ iDs}

Nawaf Al-Maharik - https://orcid.org/0000-0002-7014-6190

Peer Kirsch - https://orcid.org/0000-0002-9024-7933

Alexandra M. Z. Slawin - https://orcid.org/0000-0002-9527-6418

David O'Hagan - https://orcid.org/0000-0002-0510-5552

\section{References}

1. Kirsch, P. Modern Fluoroorganic Chemistry, 2nd ed.; Wiley-VCH: Weinheim, Germany, 2013. doi:10.1002/9783527651351
2. Kirsch, P.; Bremer, M. Angew. Chem., Int. Ed. 2000, 39, 4216-4235. doi:10.1002/1521-3773(20001201)39:23<4216::aid-anie4216>3.0.co;2$\mathrm{k}$

3. Hird, M. Chem. Soc. Rev. 2007, 36, 2070-2095. doi:10.1039/b610738a

4. O'Hagan, D. Chem. Soc. Rev. 2008, 37, 308-319. doi:10.1039/b711844a

5. Bremer, M.; Kirsch, P.; Klasen-Memmer, M.; Tarumi, K. Angew. Chem., Int. Ed. 2013, 52, 8880-8896. doi:10.1002/anie.201300903

6. Kirsch, P.; Tarumi, K. Angew. Chem., Int. Ed. 1998, 37, 484-489. doi:10.1002/(sici)1521-3773(19980302)37:4<484::aid-anie484>3.0.co; 2-6

7. Kirsch, P.; Heckmeier, M.; Tarumi, K. Liq. Cryst. 1999, 26, 449-452. doi:10.1080/026782999205236

8. Bremer, M.; Lietzau, L. New J. Chem. 2005, 29, 72-74. doi:10.1039/b414312d

9. Al-Maharik, N.; Kirsch, P.; Slawin, A. M. Z.; Cordes, D. B.; O'Hagan, D. Org. Biomol. Chem. 2016, 14, 9974-9980. doi:10.1039/c6ob01986b

10. Yamada, S.; Hashishita, S.; Konishi, H.; Nishi, Y.; Kubota, T.; Asai, T.; Ishihara, T.; Konno, T. J. Fluorine Chem. 2017, 200, 47-58. doi:10.1016/j.jluchem.2017.05.013

11. Fang, Z.; Cordes, D. B.; Slawin, A. M. Z.; O'Hagan, D. Chem. Commun. 2019, 55, 10539-10542. doi:10.1039/c9cc05749h

12. Hruschka, S.; Fröhlich, R.; Kirsch, P.; Haufe, G. Eur. J. Org. Chem. 2007, 141-148. doi:10.1002/ejoc.200600579

13. Thomson, C. J.; Zhang, Q.; Al-Maharik, N.; Bühl, M.; Cordes, D. B.; Slawin, A. M. Z.; O'Hagan, D. Chem. Commun. 2018, 54, 8415-8418. doi:10.1039/c8cc04964e

14. Wang, F.; Luo, T.; Hu, J.; Wang, Y.; Krishnan, H. S.; Jog, P. V.; Ganesh, S. K.; Prakash, G. K. S.; Olah, G. A. Angew. Chem., Int. Ed. 2011, 50, 7153-7157. doi:10.1002/anie.201101691

15. Meyer, O. G. J.; Fröhlich, R.; Haufe, G. Synthesis 2000, 1479-1490. doi:10.1055/s-2000-7103

16. Bosch, M.; Schlaf, M. J. Org. Chem. 2003, 68, 5225-5227. doi:10.1021/jo034376h

17. Conner, M. L.; Brown, M. K. J. Org. Chem. 2016, 81, 8050-8060. doi:10.1021/acs.joc.6b01446

18. Dewar, M. J. S.; Zoebisch, E. G.; Healy, E. F.; Stewart, J. J. P. J. Am. Chem. Soc. 1985, 107, 3902-3909. doi:10.1021/ja00299a024

\section{License and Terms}

This is an Open Access article under the terms of the Creative Commons Attribution License (http://creativecommons.org/licenses/by/4.0). Please note that the reuse, redistribution and reproduction in particular requires that the authors and source are credited.

The license is subject to the Beilstein Journal of Organic Chemistry terms and conditions: (https://www.beilstein-journals.org/bjoc)

The definitive version of this article is the electronic one which can be found at: $\underline{\text { doi: } 10.3762 / \text { bjoc. } 16.65}$ 